\title{
SISTEMA DE PRÁCTICAS VIRTUALES COMO ALTERNATIVA AL LABORATORIO PRESENCIAL EN ASIGNATURAS DE INGENIERÍA DE CONTROL
}

\author{
Michelena Grandío, Álvaro ${ }^{1}$; Zayas-Gato, Francisco ${ }^{1}$; Jove, Esteban ${ }^{1}$; Casteleiro-Roca \\ José-Luis ${ }^{1}$; Arce Fariña, María Elena ${ }^{1}$; Quintián, Héctor ${ }^{1}$; Calvo-Rolle, José Luis ${ }^{1}$ \\ ${ }^{1}$ Universidade da Coruña, \\ Departamento de Ingeniería Industrial
}

\section{RESUMEN}

El principal reto en los sistemas de educación superior en los dos últimos años, a raíz de la pandemia mundial de COVID-19, ha sido el poder mantener la calidad de la educación impartida con las restricciones de movilidad y aforos impuestas por las autoridades. Es por el ello que la transformación hacia actividades docentes no presenciales supone uno de los principales objetivos del sistema educativo. Con todo ello en mente, en este artículo se presenta una experiencia docente basada en la virtualización de procesos industriales y la aplicación de sistemas de control en el marco de prácticas de asignaturas de ingeniería de control en tiempo real. Para poder logarlo y además disponer de un sistema con gran realismo, se han integrado los softwares de virtualización de componentes "Factory I/O", junto con softwares de control como "Matlab" a través de Node-RED, todo ello en tiempo real. Con esto, se consigue emular con gran realismo las plantas de control de nivel disponibles en los laboratorios de la Escuela Universitaria Politécnica de la Universidade da Coruña.

PALABRAS CLAVE: Laboratorio virtual, prácticas de control y docencia no presencial. 


\section{CITA RECOMENDADA:}

Michelena Grandío, Álvaro; Zayas Gato, Francisco; Jove, Esteban; Casteleiro Roca José Luis; Arce Fariña, María Elena; Quintián, Héctor; Calvo Rolle, José Luis (2021): Sistema de prácticas virtuales como alternativa al laboratorio presencial en asignaturas de Ingeniería de Control. En García Naya, J.A. (ed.) (2021). Contextos universitarios transformadores: a nova normalidade académica. Leccións aprendidas e retos de futuro. V Xornadas de Innovación Docente. Cufie. Universidade da Coruña. A Coruña (pág. 249-259).

DOl capítulo: https://doi.org/10.17979/spudc.9788497498180.249

DOI libro: https://doi.org/10.17979/spudc.9788497498180

\section{ABSTRACT}

The main challenge in higher education systems in the last two years, as a result of the global COVID-19 pandemic, has been to be able to maintain the quality of the education provided with the mobility and capacity restrictions imposed by the authorities. That is why the transformation towards non-face-toface teaching activities is one of the main objectives of the educational system. With all this in mind, this article presents a teaching experience based on the virtualization of industrial processes and the application of control systems within the framework of real-time control engineering subjects. In order to achieve this and also have a system with great realism, "Factory $1 / 0$ " component virtualization software has been integrated, along with control software such as "Matlab" through Node-RED, all in real time. With this, it is possible to emulate with great realism the level control plants available in the laboratories of the Polytechnic University School of the University of A Coruña.

KEY WORDS: Virtual laboratory, control practices and non-face-to-face teaching 


\section{INTRODUCCIÓN}

En los últimos dos años, debido a la pandemia mundial ocasionada por el COVID-19, la enseñanza universitaria se ha visto drásticamente afecta. Uno de los principales efectos ha sido un incremento notable en la docencia semi-presencial o la no presencial. Si bien en algunas disciplinas es relativamente sencillo esta modificación del tipo de docencia, en el caso de las prácticas en las disciplinas de ingeniería industrial, este tipo de docencia no es viable 0 bien es compleja.

Los equipos de los que se dispone en los laboratorios suelen ser escasos debido a los costes que conlleva, y además difícilmente accesibles de forma online, para poder adaptar las prácticas a una docencia no presencial. En la actualidad los grupos de trabajo se han reducido 0 incluso han sido prohibidos para prevenir la transmisión del virus. De ahí que muchos profesores se esforzaran por rediseñar sus métodos de enseñanza, apoyándose en recursos tecnológicos para ofrecer una alternativa a los laboratorios reales $[8,10,11]$.

A día de hoy existen diversas herramientas de digitalización para la implementación de entornos virtuales $[3,4,5,7,13]$, sin embargo suelen ser sistemas cerrados con los que no es sencillo integrar otros softwares que se usan en los laboratorios. En este contexto, se propone la integración de una herramienta software de virtualización de entornos industriales con otra de programación y control empleada en gran cantidad de laboratorios de ingeniería a través del sistema Node-RED. En concreto se ha implementado dicho sistema en la virtualización de las plantas de control de nivel reales del Laboratorio de Optimización y Control de la Escuela Universitaria Politécnica de la Universidade da Coruña (UDC). 


\section{DESCRICIÓN DE LA EXPERIENCIA}

Durante el curso académico 2020-2021, se realizó la virtualización de una de las plantas de del Laboratorio de Optimización y Control, uno de los laboratorios de la Escuela Universitaria Politécnica de la UDC. En concreto la planta de laboratorio elegida fue el sistema de control de nivel en depósitos, ya que el principal problema de dichas plantas es el escaso número de las mismas, lo que dificulta la organización de las prácticas debido a las restricciones de compartición de materiales por el COVID-19 [1, 2, 6, 9, 12].

\subsection{Sistema elegido para su virtualización}

El sistema real (ver Figura 1) tiene dos depósitos a diferentes niveles. El objetivo que se persigue en las prácticas es controlar el nivel de agua en el depósito superior. Para ello se emplea una bomba centrífuga que impulsa el agua del depósito inferior al superior. Ambos depósitos están además conectados a través de una válvula manual y otra servo pilotada que permiten la descarga del depósito superior al inferior. La medición del nivel de agua del depósito superior se realiza a través de un sensor de ultrasónico. Por último, el control de la bomba de agua se realiza con un variador de velocidad.

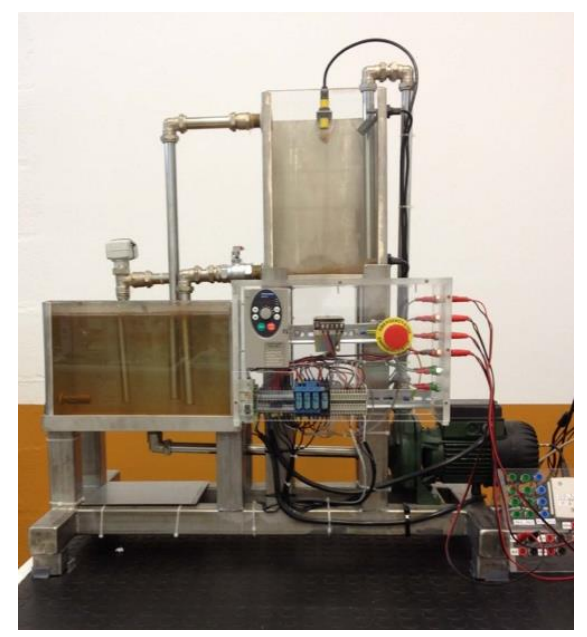

Figura 1. Planta de control de nivel 
Todo el sistema tiene una única entrada, potencia a la bomba y una única salida, el nivel de agua, ambas son señales de 0-10V. Para realizar la comunicación con el ordenador donde se realizan las prácticas con diversos softwares de control de procesos, se emplea como tarjeta de adquisición una placa Arduino UNO. La Figura 2 muestra en el esquema completo de la planta junto con el flujo de información para su control.

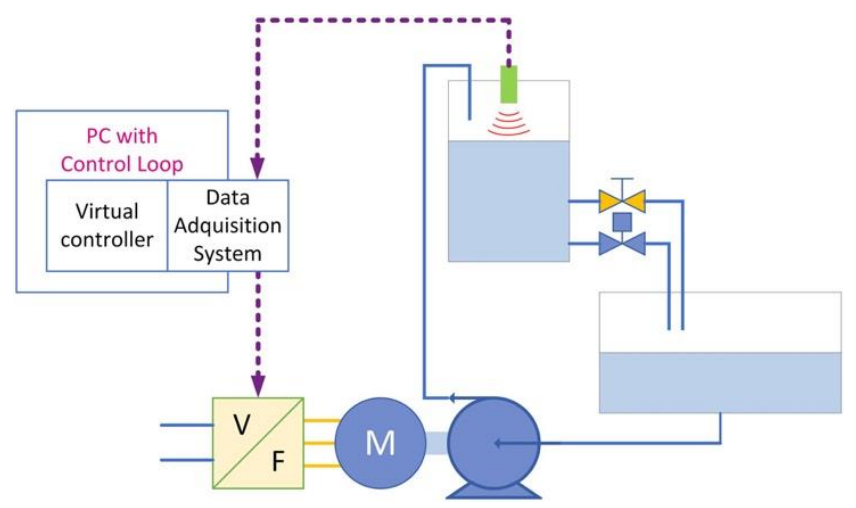

Figura 2. Esquema simplificado de la planta de control de nivel

\subsection{Integración de herramientas de virtualización y control de procesos industriales}

El primer punto a desarrollar consiste en la virtualización de la planta real de nivel explicada en el punto anterior. Para ello se emplea el software Factory $1 / 0$.

Factory $\mathrm{I} / 0$ dispone de muchos de los elementos más comunes de la industria modelados y virtualizados, para configurar un proceso industrial específico y así construir una fábrica virtual. Además, cuenta con una amplia gama de protocolos de comunicación industrial, permitiendo conexiones con PLC, microcontroladores, Ordenadores de Placa Única (SBC, Single Board Computer) o incluso con otro tipo de aplicaciones de escritorio. El resultado de la virtualización de la planta de nivel se muestra en la Figura 3.

El principal problema que plantea Factory $\mathrm{l} / 0$, es que la planta virtual de control de nivel cuenta con sensores y actuadores con entradas y salidas analógicas industriales. Sin embargo, estas señales de entrada y salida no están disponibles por hardware. Por lo tanto, una interfaz entre 
la planta virtual y el controlador real no es posible utilizando una tarjeta de adquisición de datos hardware. Por ello no sería posible su integración directa con los softwares de control de procesos empleados en prácticas como pueden ser Matlab.

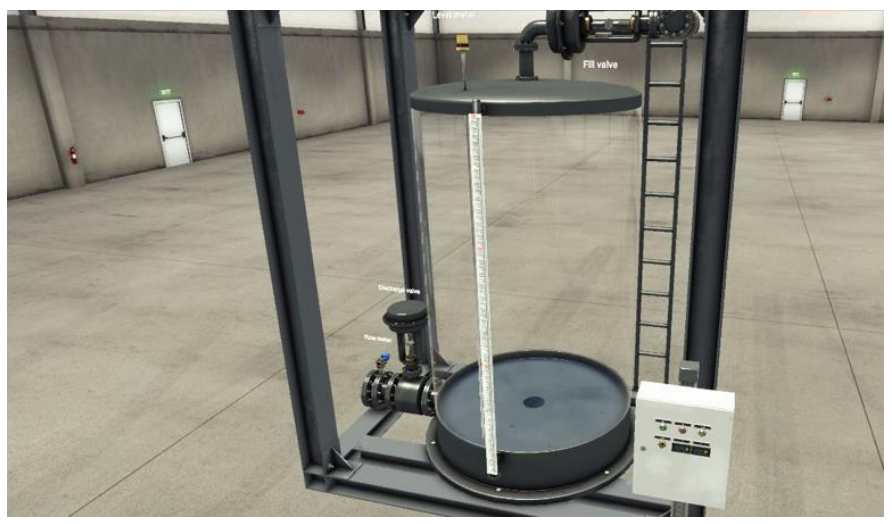

Figura 3. Planta de nivel con Factory $1 / 0$

Para poder realizar dicha integración es necesaria, entonces, una interfaz de comunicación industrial. Factory l/0 tiene varios controladores de comunicación estándar para leer y escribir las señales de entrada y salida generadas por los sistemas virtuales, siendo Modbus TCP Client y Modbus TCP Server unas de las más empleadas en los sistemas industriales reales.

Para poder implementar dicha interfaz de comunicación se emplea Node-RED, que es un software de programación gráfica para conectar dispositivos de hardware y también establecer comunicaciones entre aplicaciones. Node-RED es un sistema de programación gráfica basado en nodos, donde cada nodo dentro de la aplicación recibe datos a través de sus entradas, ejecuta acciones y envía datos a sus salidas (Figura 4).

La interfaz de comunicación a través de Node-RED se implementará mediante el protocolo de comunicación Modbus TCP para realizar el intercambio de datos. Por lo tanto, las señales de control y variables de proceso del controlador y la planta virtual se pueden enviar a través de este enlace. En este caso, el lado del cliente Modbus lo realiza la aplicación Node-RED y el 
lado del servidor por la planta virtual de nivel lo haría Factory l/0, como se puede ver en la Figura 5.

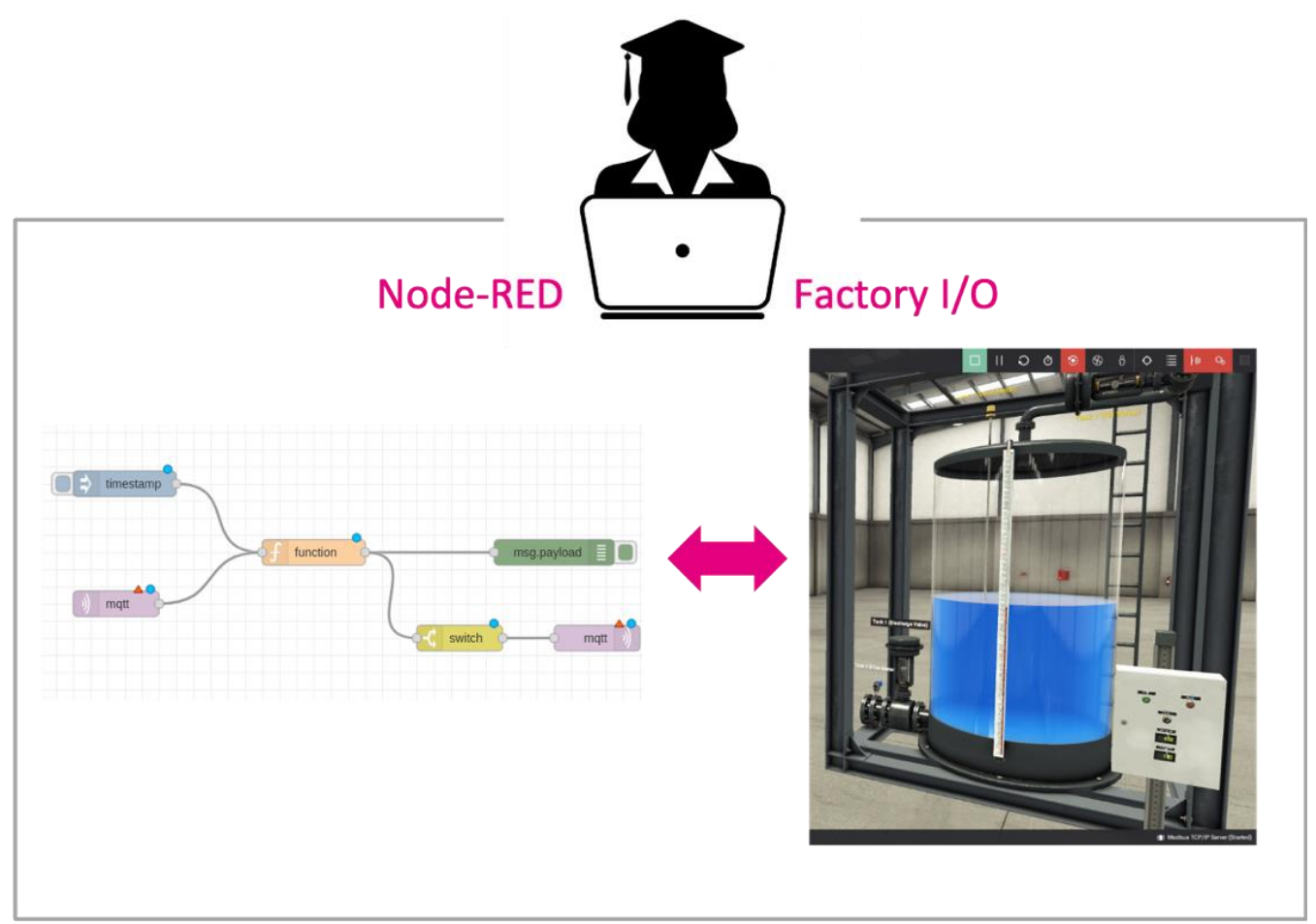

Figura 4. Entorno de programación de Node-RED

Node-RED

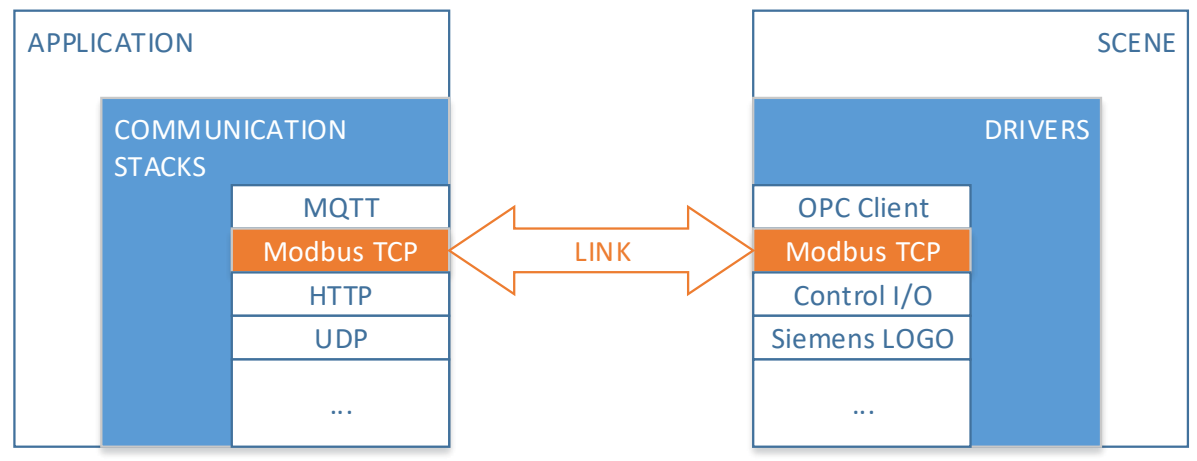

Figura 5. Enlace de comunicación entre Node-RED y Factory l/0 


\section{RESULTADOS}

Una vez virtualizada la planta de control de nivel, mediante Factory $1 / 0$, se emplea Node-RED como interfaz de comunicación con Matlab, donde se ha implementado una de las prácticas de laboratorio de la asignatura de Ingeniería de Control, que consiste en el desarrollo de un regulador PID.

La visualización de la respuesta de la planta virtual es un aspecto clave. De hecho, uno de los temas de la ingeniería de control es el ajuste empírico de los controladores PID en función de los parámetros de respuesta en el dominio del tiempo. La respuesta del sistema mediante la aplicación del sistema de control se puede ver en la Figura 6.

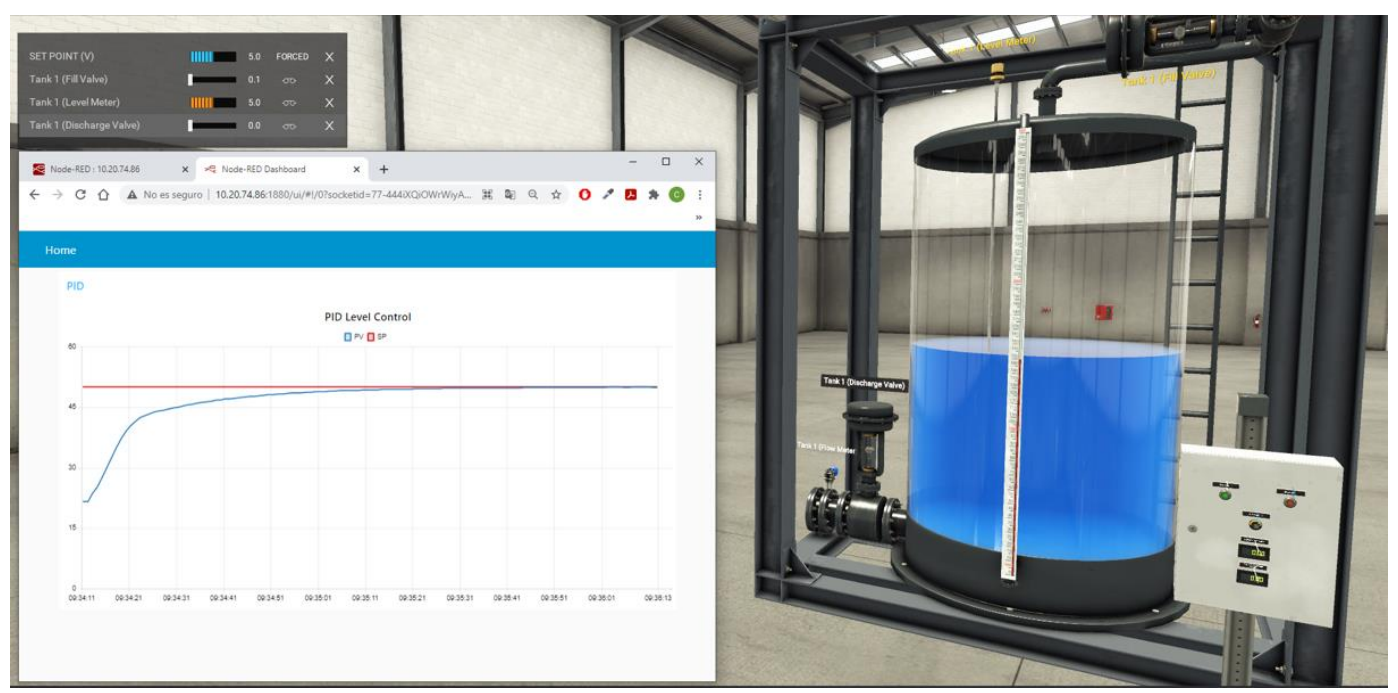

Figura 6. Integración de Node-RED y Factory I/0

Por último, una vez se comprobó la viabilidad del sistema desarrollado, este fue testado durante el curso 2020-2021 en una de las asignaturas del máster de "Informática Industrial y Robótica". Donde, a través de Factory $1 / 0$, se pudieron realizar las mismas prácticas que tendrían que hacerse de forma presencial y con necesidad de plantas industriales, de forma virtual, con ello cada alumno, de forma individual, disponía de todos los recursos necesarios 
en su propio ordenador y, además, se puede trabajar sobre las prácticas de nuevo en cualquier momento y lugar.

El feedback de los alumnos fue muy positivo, destacando en gran medida la flexibilidad que les aportaba esta nueva metodología de prácticas que, además, podía ser complementada con el trabajo sobre las plantas reales.

\section{CONCLUSIONES}

En esta experiencia docente, se ha presentado un nuevo sistema que integra dos herramientas software que permiten desarrollar prácticas de forma no presencial con un alto grado de realismo. Además, permite configurar diversos escenarios que representan diversos entornos industriales, aportando una mayor flexibilidad que en el caso de las prácticas presenciales, están limitadas por el número y tipo de procesos industriales físicos reales disponibles en los laboratorios.

Dada la similitud entre el sistema virtual con el caso real mostrado en la solución implementada, se puede concluir que esta propuesta se presenta como un método interesante para evitar un cambio mayor en la programación de las prácticas de laboratorio como consecuencia de la pandemia COVID-19.

Después de validar la solución propuesta, podemos concluir que se han obtenido muy buenos resultados. Además, se ha logrado una gran aceptación por parte de estudiantes y profesores.

Como trabajos futuros, la virtualización de otras plantas de laboratorio y sistemas como lazos de control de temperatura, podrían ayudar a mitigar el impacto de la pandemia en la modalidad presencial. Además, el uso en otras asignaturas, e incluso su uso de forma transversal entre varias asignaturas podrían enriquecer en gran medida la calidad de la enseñanza en las disciplinas de ingeniería. 


\section{REFERENCIAS}

Casteleiro-Roca, J.L., Barragán, A.J., Segura, F., Calvo-Rolle, J.L., Andújar, J.M.: Fuel cell output current prediction with a hybrid intelligent system. Complexity (2019)

Crespo-Turrado, C., Casteleiro-Roca, J.L., Sánchez-Lasheras, F., López-Vázquez, J.A., De Cos Juez, F.J., Pérez Castelo, F.J., Calvo-Rolle, J.L., Corchado, E.: Comparative study of imputation algorithms applied to the prediction of student performance. Logic Journal of the IGPL 28(1), 58-70 (2020)

Daniel, J.: Education and the covid-19 pandemic. Prospects 49(1), 91-96 (2020)

Fernández-Serantes, L.A., Vázquez, R.E., Casteleiro-Roca, J.L., Calvo-Rolle, J.L., Corchado, E.: Hybrid intelligent model to predict the soc of a Ifp power cell type. In: International conference on hybrid artificial intelligence systems. pp. 561-572. Springer (2014)

Jove, E., Casteleiro-Roca, J.L., Quintián, H., Méndez-Pérez, J.A., Calvo-Rolle, J.L.: Outlier generation and anomaly detection based on intelligent one-class techniques over a bicomponent mixing system. In: International Workshop on Soft Computing Models in Industrial and Environmental Applications. pp. 399-410. Springer (2019)

Jove, E., Casteleiro-Roca, J.L., Quintián, H., Méndez-Pérez, J.A., Calvo-Rolle, J.L.: Virtual sensor for fault detection, isolation and data recovery for bicomponent mixing machine monitoring. Informatica 30(4), 671-687 (2019)

Jove, E., Casteleiro-Roca, J.L., Quintián, H., Méndez-Pérez, J.A., Calvo-Rolle, J.L.: A new method for anomaly detection based on non-convex boundaries with random twodimensional projections. Information Fusion 65, 50-57 (2021)

Jove, E., Casteleiro-Roca, J.L., Quintián, H., Simic, D., Méndez-Pérez, J.A., Luis Calvo-Rolle, J.: Anomaly detection based on oneclass intelligent techniques over a control level plant. Logic Journal of the IGPL 28(4), 502-518 (2020)

Laal, M., Laal, M.: Collaborative learning: what is it? Procedia-Social and Behavioral Sciences 31, 491-495 (2012) 
Nicola, M., Alsa, Z., Sohrabi, C., Kerwan, A., Al-Jabir, A., Iosifidis, C., Agha, M., Agha, R.: The socio-economic implications of the coronavirus and covid-19 pandemic: a review. International journal of surgery (2020)

Pérez-López, E., Atochero, A.V., Rivero, S.C.: Educación a distancia en tiempos de covid-19: Análisis desde la perspectiva de los estudiantes universitarios. Revista Iberoamericana de Educación a Distancia 24(1), 331-350 (2021)

Ray, S., Srivastava, S.: Virtualization of science education: a lesson from the covid-19 pandemic. Journal of proteins and proteomics 11, 77-80 (2020)

Vasiliadou, R.: Virtual laboratories during coronavirus (covid-19) pandemic. Biochemistry and Molecular Biology Education 48(5), 482-483 (2020) 
